\title{
Effect of incorporating white, red or black quinoa flours on free and bound polyphenol content, antioxidant activity and colour of bread
}

\section{Jaime Ballester-Sánchez ${ }^{2}$ Jose Vicente Gil ${ }^{1,2}$ - Claudia Monika Haros ${ }^{2}$ - María Teresa Fernández-Espinar ${ }^{2} *$}

${ }^{1}$ Departamento de Medicina Preventiva y Salud Pública, Ciencia de Alimentos, Toxicología y Medicina Forense, Universidad de Valencia, Spain; ${ }^{2}$ Instituto de Agroquímica y Tecnología de Alimentos (IATA),Consejo Superior de Investigaciones Científicas (CSIC), Av. Agustín Escardino 7, Parque Científico, 46980 Paterna, Valencia, Spain. * Corresponding author, Phone: +34 9639000 22; Fax: +34 9636363 01; e-mail: tfer@iata.csic.es (María Teresa Fernández-Espinar)

\begin{abstract}
Interest in quinoa as a functional food ingredient is currently emerging. The flours from white, red and black quinoa seeds were analysed in terms of total polyphenol content and antioxidant activity. They were incorporated at $25 \%$ on flour basis into the bread dough formula to evaluate their potential to improve the functional properties of wheat breads. The contribution of extractable polyphenols (soluble forms) and the largely unexplored hydrolysable polyphenols (bound forms that can be found in the residues of the former) were taken into account to reflect a realistic healthpromoting potential of breads. The red and black quinoa varieties stood out compared to wheat flour, with about double the polyphenol content and up to 4.7-fold increments in antioxidant activity when considering the sum of extractable and hydrolysable polyphenols. The red and black flours were equally effective in intensifying the antioxidant properties of bread despite the baking process (between 2- and 3-fold). They produced significant changes in the parameters that describe crust and crumb colour ( $\left.\mathrm{L}^{*}, \mathrm{a}^{*}, \mathrm{~b}^{*}\right)$. A clear darkening was observed compared to the control bread, an appealing attribute for lovers of unconventional and natural products. According to our results,
\end{abstract}


the flours from the coloured quinoa seeds could be considered interesting antioxidant sources and be applied as natural ingredients in bread-making; new, promising and valuable unconventional products for consumers and producers could be developed.

\section{Keywords}

Quinoa flours (white, red and black) • Bread • Free and bound polyphenols · Total polyphenol content $\cdot$ antioxidant activity $\cdot$ Colour

\section{Abbreviations}

AC

Antioxidant capacity

TAC

Total Antioxidant capacity

EPF Extractable polyphenol fraction

HPF Hydrolysable polyphenol fraction

PC Polyphenol content

TPC Total polyphenol content

\section{Introduction}

Eating products with antioxidant properties has been popularised due to the belief that some dietary patterns can be useful for preventing certain pathological conditions with a positive effect on people's quality of life [1]. The food industry has reacted to this market opportunity and has shown interest in developing new products with improved antioxidant properties for years. The use of antioxidants of plant-based ingredients has become important in most food innovations as a way to obtain current consumers' confidence, who feel especially attracted by everything that is organic and natural.

Ancient grains are perceived by consumers as being more healthy and natural compared to common cereals. They have aroused much interest as a source of ingredients to develop new functional foods [2]. Quinoa (Chenopodium quinoa Willd.), a pseudocereal from South America, has become very popular and well appreciated, which is not surprising given its remarkable nutrition composition 
and other interesting attributes [3]. Quinoa is also recognised as an excellent source of polyphenols. Health benefits associated with its intake have been described, especially for lowering the risk of oxidative stress-related diseases $[4,5]$.

Bread is regularly and widely consumed, and is considered traditionally important for human nutrition. Research shows this food matrix as an appropriate vehicle to introduce bioactive compounds in diets deficient in antioxidants, and the resulting breads are very much in demand [6]. Quinoa is a relatively new ingredient in bread, and its impact on polyphenol and antioxidant activity contents has barely been addressed [7, 8, 9]. Addition of quinoa flour generally increases total polyphenol content and antioxidant activity in the resulting breads.

The present work assesses in-depth the potential interest of incorporating quinoa flour into bread and the changes that occur during the baking process in terms of colour and antioxidant properties related to total polyphenol content, specifically: 1) three types of quinoa (white, red and black Organic quinoa Real(C) were used to identify whether a specific variety was more appropriate as an antioxidant source; 2) the contribution of extractable (soluble forms) and hydrolysable polyphenols (bound forms that can be found in the residues of the former) to the total polyphenol content and antioxidant activity was contemplated to reflect a more realistic health-promoting potential of breads. The aforementioned studies [7, 8, 9] ignore the hydrolysable polyphenol content of quinoa breads despite the presence of this fraction being appreciable in plant food and, therefore, relevant in dietary intake [10]. These compounds seem to exert some biological activity in the colon by contributing to health properties with soluble polyphenols [10]; 3) the association between colour and phenolic content in seeds and breads was also analysed.

\section{Materials and Methods}

\section{Materials}


White, red and black quinoa seeds (Organic quinoa Real ${ }^{\odot}$ ), commercially available from ANAPQUI (La Paz, Bolivia), were purchased from Ekologikoak (Bizkaia, Spain). Quinoa seeds were ground separately to obtain the corresponding flour in a commercial coffee blender (Aromatic, Taurus, Oliana, Spain). Wheat flour was purchased from a local market and dehydrated yeast (Saccharomyces cerevisiae, Maizena, Spain) was used as a starter for the breadmaking process.

\section{Bread-making procedure}

Control bread and three types of quinoa breads containing flour of each quinoa variety (white, red and black) were produced. Wheat flour was replaced by quinoa flour at $25 \mathrm{~g} / 100 \mathrm{~g}$ of flour. This quinoa concentration was stablished as optimal to bread performance and acceptance by consumers in a previous work [11]. A detailed description can be found in supplementary material (ESM1).

\section{Colour assessment}

Colour was measured by a digital colorimeter (Chroma Meter CR-400, Konika Minolta Sensing, Japan). Colour differences were recorded as CIELab, L* (lightness), a* (redness to greenness) and $\mathrm{b}^{*}$ (yellowness to blueness) values. $\Delta \mathrm{E}^{*}=\left[\left(\Delta \mathrm{L}^{*}\right) 2+\left(\Delta \mathrm{a}^{*}\right) 2+\left(\Delta \mathrm{b}^{*}\right) 2\right] 1 / 2$. Each sample was measured 4 times at different points due to heterogeneity from the quinoa.

\section{Extraction of extractable and hydrolysable phenolic compounds}

The extractable polyphenols fractions (EPF) and the hydrolysable polyphenols fractions (HPF) were obtained following the method of Saura-Calixto et al. [12]. A more detailed description can be found in supplementary material (ESM1).

\section{Polyphenol content}

Polyphenol content (PC) was determined in both the EPF and HPF by the method of FolinCiocalteu [13]. Total polyphenol content (TPC) was determined as the sum of PC in both the EPF and HPF. A more detailed description can be found in supplementary material (ESM1).

\section{Determination of antioxidant capacity}


The antioxidant capacity (AC) was determined in both the EPF and HPF by two spectrophotometric assays: DPPH ( $\alpha$-diphenyl- $\beta$-picrylhydrazyl free radical scavenging method) according to BrandWilliams et al. [14] and FRAP (ferric reducing antioxidant power assay) according to Benzie and Strain [15]. Total antioxidant activity (TAC) was determined as the sum of AC in both the EPF and HPF. A more detailed description can be found in supplementary material (ESM1).

\section{Betalains content}

Betalains were quantified in the EPF of the quinoa flours with a microplate spectrophotometer reader (Power Wave HT, BioTek Instruments, Winooski, VT, USA) at two wavelengths (480 and $536 \mathrm{~nm}$ ). Betalain concentration was evaluated as described by [16]. Determinations were made in triplicate in each extract and the results were expressed as mg / $100 \mathrm{~g} \mathrm{d.m.}$

\section{Statistical analysis}

ANOVA, followed by the Fisher's Least Significant Differences (LSD) test, was used to compare PC, TPC, AC, TAC of polyphenol extracts as well as the colour values of flours, breads or seeds. Pearson's correlation analysis was used to show the relationship between various parameters. The significance level was set in both cases at $p<0.01$ and calculations were made using the IBM SPSS Statistics software, v22 for Windows. The pairwise comparisons between the means of the estimated and calculated values before and after baking, respectively, were performed by a Student's t-test (Microsoft Excel 2010). $p$ values <0.05 were considered statistically significant.

\section{Results and Discussion}

\section{Polyphenol content and antioxidant activity in the EPF and HPF of flours}

PC and AC were determined in the EPF and HPF of flours. TPC (PC in EPF + PC in HPF) and TAC (AC in EPF + AC in HPF) were considered for complete contribution estimation (Table 1, online resource). The PC of the EPF in the black quinoa was significantly higher than in the other varieties ( $p<0.01$ ) (around 1.3-fold). Regarding the PC of the HPF, the red and black quinoas were 
indistinguishable $(p>0.01)$ and stood out compared to the colouredless variety $(p<0.01)$. The AC determinations revealed that the white quinoa presented significantly lower FRAP and DPPH ( $p<$ 0.01) values in both the EPF and HPF fractions compared to the coloured varieties, which followed the same pattern as in the polyphenols content. DPPH and FRAP values in the EPF of the black variety were significantly higher than in the red one $(p<0.01)$. Values in the HPF were not consistent; both dark varieties were indistinguishable by DPPH ( $\mathrm{p}>0.01$ ) while FRAP values in the black quinoa flour were significantly lower compared to those of the red variety $(\mathrm{p}<0.01)$.

The determination of PC and AC of the quinoa grains has been reported (references can be found in review articles on the subject [17]). The shown values varied considerably, due mainly to differences in the employed extraction procedure and analytical protocols used. To compare the polyphenol values that corresponded to the EPF, those works that used the same extractive protocol as ours (methanol/water followed by acetone/water) were chosen. Our PC values were slightly higher, i.e. 5.03-6.60 mg/g vs. 1.23-3.41 mg/g [18], 1.44-2.1 mg/g [8], 3.75 mg/g [19] and 4 mg/g [20]. Regarding AC, the DPPH and FRAP values for the white quinoa (3.37 and $2.7 \mathrm{mg} / \mathrm{g}$, respectively) agreed with those described by Pellegrini et al. [20] and Brend et al. [8], who reported lower values for the coloured varieties (between 1.6- and 3-fold lower).

The PC and AC values that we obtained in the HPF were also compared with those reported in the literature, although data published on the HPF in quinoa are scarce [18, 21]. The obtained PC values were much higher than those described in these works, with up to 7-fold increments between the white quinoa varieties, and about 4-fold ones between the coloured ones. Regarding AC, our DPPH and FRAP values were also higher than other reported ones [21], around 2- to 9-fold higher. The fact that these authors used much shorter hydrolysis times compared to our assay conditions could be the reason for the less efficient release documented.

When considering the final contribution of both the EPF and HPF to TPC and TAC (EPF plus HPF), both coloured varieties presented the same behaviour and stood out from the white quinoa. 
This clear trend between higher phenolic content and antioxidant activity values and the darker varieties has been previously described $[8,21]$. The PC values associated with HPF were much higher in the flours of the three varieties than the values in the EPF (they dobled the white quinoa, and were 4.3- and 3.6-fold more for the red quinoa and the black quinoa, respectively), which constituted between $70 \%$ and $82 \%$ of the TPC and TAC (EPF + HPF). This remarkable contribution of HPF is consistent with that described in the literature for others foods and raw materials, and suggests the importance of not underestimating this polyphenol fraction [10].

As our main objective was to include quinoa in bakery products, the TPC and TAC of the three flours were compared to those of wheat. When considering the EPF + HPF sum, the red and black quinoa flours stood out for their significantly higher TPC and TAC values than the wheat flour (around twice and up to 4.7-fold increments, respectively), whereas the latter was indistinguishable from the white quinoa. The PC values in the EPF were significantly higher in the wheat flour compared to the white and red quinoa flours $(p<0.01)$, and equalled to the black variety $(p>0.01)$. The AC analysis in these extracts showed that the wheat flour presented clearly lower AC than the quinoa flours $(p<0.01)$. These contradictory results suggest an overestimation of the value of polyphenols in the wheat flour EPF extract through the interaction of compounds other than phenols with the Folin-Ciocalteu reagent, as mentioned elsewhere [22].

\section{Colour analysis of flours and seeds. Relationship with phenolic contents}

The colour parameters of flour and seeds were also determined, and differences between both quinoa form presentations were observed (Table 2, online resource). The colour of the quinoa flours was a combination of yellowness $\left(b^{*}\right)$, redness $\left(a^{*}\right)$ and lightness $\left(\mathrm{L}^{*}\right)$, except for the white quinoa flour that showed a negative $a^{*}$ parameter related with a greenish tint. $\mathrm{L}^{*}$ and $\mathrm{a}^{*}$ statistically differed among all the quinoa flours and, as expected, white quinoa flour $\mathrm{L}^{*}$ and red quinoa flour a* had the highest values; $b^{*}$ was the equivalent in the white and red flours and was higher than in the 
black one. In terms of total colour differences $\left(\Delta \mathrm{E}^{*}\right)$ in relation to wheat flour, the quinoa flours presented differences of 3.8 for the black quinoa, and of 15.5 and 19.9 for red and black ones, respectively. The colour parameters were affected by milling, which was expected given the pigment accumulation in the grain outer layer and the processing effect on colour lightening. Lightness ( $\left.\mathrm{L}^{*}\right)$ was generally higher in flours compared to seeds, and redness (a*) was lower. The black quinoa seeds obtain the lowest $\mathrm{L}^{*}$ and $\mathrm{b}^{*}$ values, as occurred for the corresponding flour, compared to the other quinoa varieties.

An association between colour parameters and phenolic content has been described in different fruits, grains and plants. In the present work, the TPC in the quinoa samples negatively and significantly correlated with the $L^{*}$ values of seeds $(r=-0.970, p=0.001)$. We now know that the different seed colourations are not really due to the polyphenolic compounds of the anthocyanins type as described by Pasko et al. [19], but to the presence of pigments called betalains with antioxidant activity properties [18, 16]. Abdherrahim et al. [18] proposed that the regulatory mechanisms involved in the biosynthesis of betalains and polyphenols respond to the same environmental conditions and, therefore, a simultaneous increase in both compounds takes place. This could explain why good correlations between both compounds were observed. Moreover, pigments are extracted in the same aqueous/organic solvents as extractable polyphenols [18]. We detected the presence of betalains in our quinoa EPFs (3.43, 6.15 and $7.19 \mathrm{mg} / 100 \mathrm{~g}$ in the white, red and black quinoa, respectively). This result agrees with the fact that the correlation found between PC and AC was lower in EPF (FRAP: $\mathrm{r}=0.610, p=0.07$; DPPH; $\mathrm{r}=0.559, p=0.16$ ) than in the HPF (FRAP: $r=0.934, p<0.0001$; DPPH; $r=0.884, p<0.0001$ ). It is indicative that betalains contribute, together with polyphenolic compounds, to the antioxidant activity of the EPF.

\section{Effect of quinoa incorporation on breads}

\section{Phenolic contents and colour}


The PCs of quinoa and the control breads were determined (Table 3, online resource). All the quinoa breads presented an equivalent PC in the EPF, around 2-fold higher than the control bread ( $p$ $<0.01$ ). However regarding PC in the HPF, only the black quinoa bread displayed statistically higher values. No significant differences were observed for the TPC values (EPF + HPF) compared to the control bread ( $p>0.01$ ), but an increasing trend in content was observed as the used quinoa was darker.

The effect of baking on phenolic content was evaluated in the quiona breads by comparing the values in flour mixtures (estimated by taking the percentage of each flour used to make doughs and the corresponding phenolic values in Table 1 "online resource") with the value determined in breads (Table 3, online resource). As Figure 1 shows, a drop between 1.5- and 1.8-fold in the PC in EPF was detected in the quinoa breads $(p<0.05)$. Contrarily, the PC result in the HPF was striking as a statistically significant increase took place after the thermal process in the white (2-fold) and black quinoa (1.7-fold) breads ( $p=0.0040$ and 0.0038 , respectively). The same behaviour and an increase of the same order were noted for the red quinoa bread, but not significantly $(p=0.0519)$. When combining both the EPF and HPF, PC became statistically higher in the white and black quinoa breads after baking. Greater thermal sensitivity of soluble polyphenols than hydrolysable ones has been described in breads containing barley flour (Holtekjolen, as cited in Dziki et al. [6]). Dziki et al. [6] reviewed the changes in PC and AC during the bread-making process. Destruction of compounds has sometimes been reported, but unaffected or new compounds seemed to form on other occasions. Reduction has been associated with either the heat instability of compounds or the formation of complexes with bread proteins and carbohydrates that make them less extractable. The formation of Maillard reaction products has been widely used to explain the increase in the concentration of phenolics, as well as the breackage of covalently bound phenolic compounds. Even an overestimation of the analytical methodology has been suggested (see Dziki et al. [6] and references therein). Thus the occurrence of different effects of baking on the TPC being influenced 
by the type of ingredient used during production cannot be ruled out (Gelinas and McKinnon as cited in Dziki et al. [6]).

The inclusion of $25 \%$ of quinoa flours influenced the final colour of breads (Table 4 , online resource). In terms of total colour differences $\left(\Delta \mathrm{E}^{*}\right)$, the three quinoa breads were significantly more coloured than the control bread for both crust and crumbs. Except for crumbs of the white quinoa, the $\Delta \mathrm{E}^{*}$ values were higher than 5 units and, therefore, indicates that differences can be visually perceptible by consumers. The $\mathrm{L}^{*}$ (lightness) values lowered for crumbs when darker varieties were incorporated. The opposite behaviour for parameter a* (redness) occurred, which increased in the following sequence: white quinoa $>$ black quinoa $>$ red quinoa. The differences for yellowing $\left(\mathrm{b}^{*}\right)$ for crumbs were less evident, but a decreasing trend was observed in parallel to darkening. In summary, the breads containing the darker flours were more reddish- and less yellowish-coloured than those with the white quinoa and the control bread. The colour parameters found in breads could be attributed to the colour caracteristic of each quinoa variety (see Table 2, online resource), but also to the formation of Maillard products (Gelinas and McKinnon as cited in Dziki et al. [6]). Parameter 100-L* has been proposed as a marker to estimate the formation of hydroxymethylfurfural (intermediate products of the Maillard reaction) [23]. The 100-L* values for quinoa bread crusts (Table 4, online resource) were significantly higher compared to the values in the corresponding flours (Table 2 , online resource) $(p<0.01)$. This was indicative of the presence of hydroxymethylfurfural and therefore, Maillard reaction products could be partly responsible for the increment in the TPC observed after baking (Fig. 1).

\section{Contribution to antioxidant properties}

In order to evaluate whether the remarkable AC detected in the quinoa flours remained after baking, this parameter was determined in both the EPF and HPF of the resulting breads, and was compared to that of the control (Fig. 2). The sum of the AC values in both the EPF and HPF (TAC) was significantly higher $(p<0.01)$ in the red and black quinoa breads than in the control (DPPH: around 
2-fold, FRAP: around 3-fold). These results showed that these two flours varieties were equally effective in intensifying the antioxidant properties of wheat bread, unlike the white quinoa bread, which was indistinguishable from the control $(p>0.01)$. In all cases, the contribution of the HPF to the sum was clearly larger than that of the EPF.

Published data about the antioxidant effect that derives from adding quinoa flour to bread formulations are scarce. Specifically, greater antioxidant activity, compared with the corresponding controls, has been described in gluten-free bread with 50\% quinoa [7] and 100\% quinoa [7, 8]. Chlopicka et al. [9] characterised the AC of the EPF from wheat bread with $15 \%$ and $30 \%$ quinoa, and they obtained DPPH and FRAP values within the range of $1.17 \mu \mathrm{mol} / \mathrm{g}$ and $73.75 \mathrm{mg}$ Trolox/100 g (i.e. $2.95 \mu \mathrm{mol} / \mathrm{g}$ ), respectively, which are of the same order as our results (DPPH: 1.41-2.17 $\mu \mathrm{mol} / \mathrm{g}$ and FRAP: 3.13-5.20 $\mu \mathrm{mol} / \mathrm{g})$. The differences they found in relation to the control bread were not as clear as those that we obtained. By respecting the HPF, we found no data about this for the quinoa breads and, as far as we know, this is the first time that a complete determination in polyphenols is provided for such a food product type. This is interesting if we take into account that both the EPF and HPF are ingested when bread is eaten.

The effect of baking conditions was evaluated by comparing AC in the flour mixtures, estimated by using the DPPH and FRAP values in Table 1 (online resource), with the experimental values in the corresponding breads (Fig. 2). Baking did not lead to any major losses in AC in the quinoa breads (Table 5, online resource). In fact we observed no modifications in the ability to quench DPPH radicals, while occasionally significant increments were noted in the FRAP values, especially for the EPF. This latter feature contradicts the post-baking losses in the PC of these fractions (see Fig. 1). The formation of the Maillard reaction products during baking is known. As they are antioxidative agents (see Dziki et al. [6] and references therein), they likely contribute to increased antioxidant activity in breads. Brend et al. [8] have described an increase in FRAP levels in 100\% red quinoa breads and referred to the same cause. 


\section{Conclusions}

The study of both the TPC and TAC of three differently coloured quinoa seeds, by considering soluble and bound fractions, indicated that this pseudocereal could be a good natural source to improve the antioxidant activity of wheat bread. The antioxidant potential was different depending on the quinoa variety used, and was strongly related with seed colour. The red and black varieties stood out and were equally effective in intensifying the antioxidant properties of wheat bread. Future research works will be needed to asses the in vivo antioxidant effect. Bread colour was affected by the colour characteristic of each quinoa variety because clear darkening was observed compared to the control bread. The bakery industry could take advantage of this attribute to attract lovers of unconventional products and consumers in general as darkness in products is associated with the un-refined products currently recommended as part of healthy lifestyle habits.

\section{Acknowledgements}

This work was financially supported by grants AGL2016-75687-C2-1-R (MEIC-Spain), PROMETEO/2017/189 (Generalitat Valenciana, Spain) and CYTED 119RT0567 (Spain). J. Ballester-Sánchez thanks the Spanish MEIC for his contract.

\section{References}

1. Langhans W (2018) Food Components in Health Promotion and Disease Prevention. J Agric Food Chem 66(10):2287-2294. https://doi.org/10.1021/acs.jafc.7b02121

2. Boukid F, Folloni S, Sforza S, Vittadini E, Prandi, B (2018) Current Trends in Ancient GrainsBased Foodstuffs: Insights into Nutritional Aspects and Technological Applications. Compr Rev Food Sci Food Saf 17(1):123-136. https://doi.org/10.1111/1541-4337.12315

3. Repo-Carrasco R, Espinoza C, Jacobsen S -E (2003) Nutritional Value and Use of the Andean Crops Quinoa (Chenopodium quinoa) and Kañiwa (Chenopodium pallidicaule). Food Rev Int 19(1-2):179-189. https://doi.org/10.1081/FRI-120018884 
4. Simnadis TG, Tapsell LC, Beck EJ (2015) Physiological effects associated with quinoa consumption and implications for research involving humans: A review. Plant Foods Hum Nutr 70(3):238-249. https://doi.org/10.1007/s11130-015-0506-5

5. Pasko P, Barton H, Zagrodzki P, Izewska A, Krosniak M, Gawlik M, Gawlik M, Gorinstein S (2010) Effect of diet supplemented with quinoa seeds on oxidative status in plasma and selected tissues of high fructose-fed rats. Plant Foods Hum Nutr 65(2):146-151. https://doi.org/10.1007/s11130-010-0164-6

6. Dziki D, Rozylo R, Gawlik-Dziki U, Swieca M (2014) Current trends in the enhancement of antioxidant activity of wheat bread by the addition of plant materials rich in phenolic compounds. Trends Food Sci Technol 40(1):48-61. https://doi.org/10.1016/j.tifs.2014.07.010

7. Álvarez-Jubete L, Wijngaard H, Arendt EK, Gallagher E (2010) Polyphenol composition and in vitro antioxidant activity of amaranth, quinoa buckwheat and wheat as affected by sprouting and baking. Food Chem 119(2):770-778. https://doi.org/10.1016/j.foodchem.2009.07.032

8. Brend Y, Galili L, Badani H, Hovav R, Galili S (2012) Total phenolic content and antioxidant activity of red and yellow quinoa (Chenopodium quinoa Willd.) seeds as affected by baking and cooking conditions. Food Nutr Sci 3:1150-1155. http://dx.doi.org/10.4236/fns.2012.38151

9. Chlopicka J, Pasko P, Gorinstein S, Jedryas A, Zagrodzki P (2012) Total phenolic and total flavonoid content, antioxidant activity and sensory evaluation of pseudocereal breads. LWT Food Sci Technol 46(2):548-555. https://doi.org/10.1016/j.lwt.2011.11.009

10. Pérez-Jiménez J, Díaz-Rubio ME, Saura-Calixto F (2013) Non-extractable polyphenols, a major dietary antioxidant: Occurrence, metabolic fate and health effects. Nutr Res Rev 26(2):118129. https://doi.org/10.1017/S0954422413000097

11. Iglesias-Puig E, Monedero V, Haros M (2015) Bread with whole quinoa flour and bifidobacterial phytases increases dietary mineral intake and bioavailability. LWT Food Sci Technol 60(1):71-77. https://doi.org/10.1016/j.lwt.2014.09.045 
12. Saura-Calixto F, Serrano J, Goñi I (2007) Intake and bioaccessibility of total polyphenols in a whole diet. Food Chem 101(2):492-501. https://doi.org/10.1016/j.foodchem.2006.02.006

13. Singleton VL, Rossi JA (1965) Colorimetry of total phenolics with phosphomolybdicphosphotungstic acid reagents. Am J Enol Vitic 16:144-158

14. Brand-Williams W, Cuvelier ME, Berset C (1995) Use of a free radical method to evaluate antioxidant activity. LWT Food Sci Technol 28:25-30. https://doi.org/10.1016/S0023$\underline{6438(95) 80008-5}$

15. Benzie I, Strain J (1996) The Ferric Reducing Ability of Plasma (FRAP) as a Measure of “Antioxidant Power: The FRAP Assay”. Anal Biochem 239:70-76. https://doi.org/10.1006/abio.1996.0292

16. Escribano J, Cabanes J, Jiménez-Atiénzar M et al (2017) Characterization of betalains, saponins and antioxidant power in differently coloured quinoa (Chenopodium quinoa) varieties. Food Chem 234:285-294. https://doi.org/10.1016/j.foodchem.2017.04.187

17. Tang Y, Tsao R (2017) Phytochemicals in quinoa and amaranth grains and their antioxidant, anti-inflammatory, and potential health beneficial effects: A review. Mol Nutr Food Res https://doi.org/10.1002/mnfr.201600767

18. Abderrahim F, Huanatico E, Segura R, Arribas S, Gonzalez MC, Condezo-Hoyos L (2015) Physical features, phenolic compounds, betalains and total antioxidant capacity of coloured quinoa seeds (Chenopodium quinoa Willd.) from Peruvian Altiplano. Food Chem 183:83-90. https://doi.org/10.1016/j.foodchem.2015.03.029

19. Pasko P, Barton H, Zagrodzki P, Gorinstein S, Folta M, Zachwieja Z (2009) Anthocyanins, total polyphenols and antioxidant activity in amaranth and quinoa seeds and sprouts during their growth. Food Chem 115(3):994-998. https://doi.org/10.1016/j.foodchem.2009.01.037 
20. Pellegrini M, Lucas-Gonzales R, Ricci A et al (2018) Chemical, fatty acid, polyphenolic profile, techno-functional and antioxidant properties of flours obtained from quinoa (Chenopodium quinoa Willd.) seeds. Ind Crops Prod 111:38-46. https://doi.org/10.1016/j.indcrop.2017.10.006

21. Tang Y, Zhang B, Xihong L, Chen PX, Zhang H, Ronghua L, Tsao R (2016) Bound phenolics of quinoa seeds released by acid, alkaline, and enzymatic treatments and their antioxidant and $\alpha$ glucosidase and pancreatic lipase inhibitory effects. J Agric Food Chem 64(8):1712-1719. https://doi.org/10.1021/acs.jafc.5b05761

22. Everette JD, Bryant QM, Green AM et al (2010) Thorough study of reactivity of various compound classes toward Folin-Ciocalteu reagent. J Agric Food Chem 58:8139-8144. https://doi.org/10.1021/jf1005935

23. Ramírez-Jiménez A, Garcia-Villanova B, Guerra-Hernandez E (2000) Hydroxymethylfurfural and methylfurfural content of selected bakery products. Food Res Int 33:833-838. https://doi.org/10.1016/S0963-9969(00)00102-2

\section{Figure captions}

Fig. 1 Effect of baking on the polyphenol content of the 25\% quinoa breads. Low-case letters refer to the comparisons of extractable (EPF) and hydrolysable polyphenol fractions (HPF); upper-case letters refer to the comparisons of the EPF + HPF sum. Comparisons were always made between flour mixtures (75\% wheat $+25 \%$ quinoa) and the bread values within each quinoa type. The bars followed by the same letter are not significantly different according to the Student's $t$-test at the 95\% confidence level. WQ: white quinoa, RQ: red quinoa, BQ: black quinoa

Fig. 2 Antioxidant activity of the extractable (EPF) and hydrolysable (HPF) polyphenol fractions from the control and the $25 \%$ quinoa breads. Low-case letters refer to the comparisons of both the EPF and HPF; upper-case letters refer to the comparisons of the EPF + HPF sum. The bars followed 
by the same letter are not significantly different according to the LSD post hoc test at the $99 \%$ confidence level

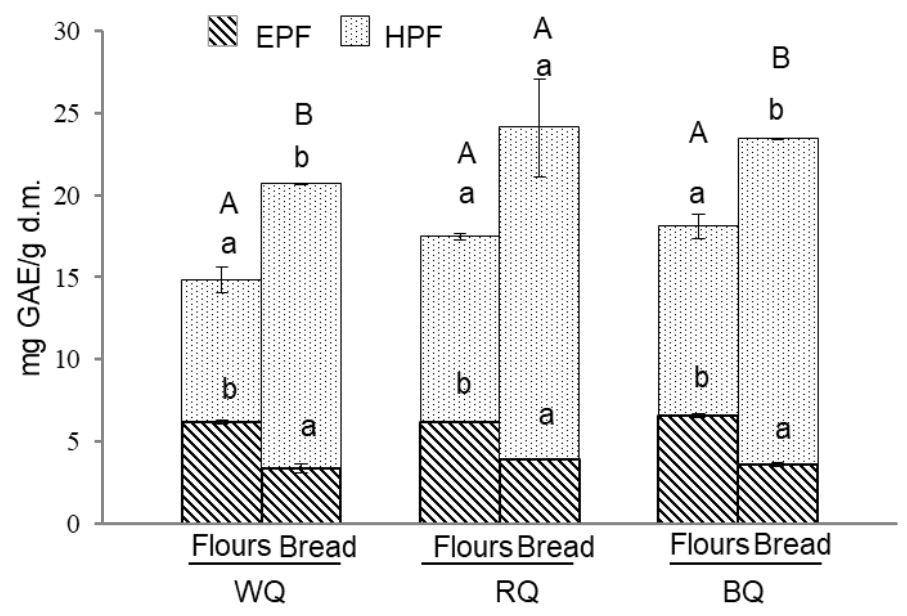

Fig. 1
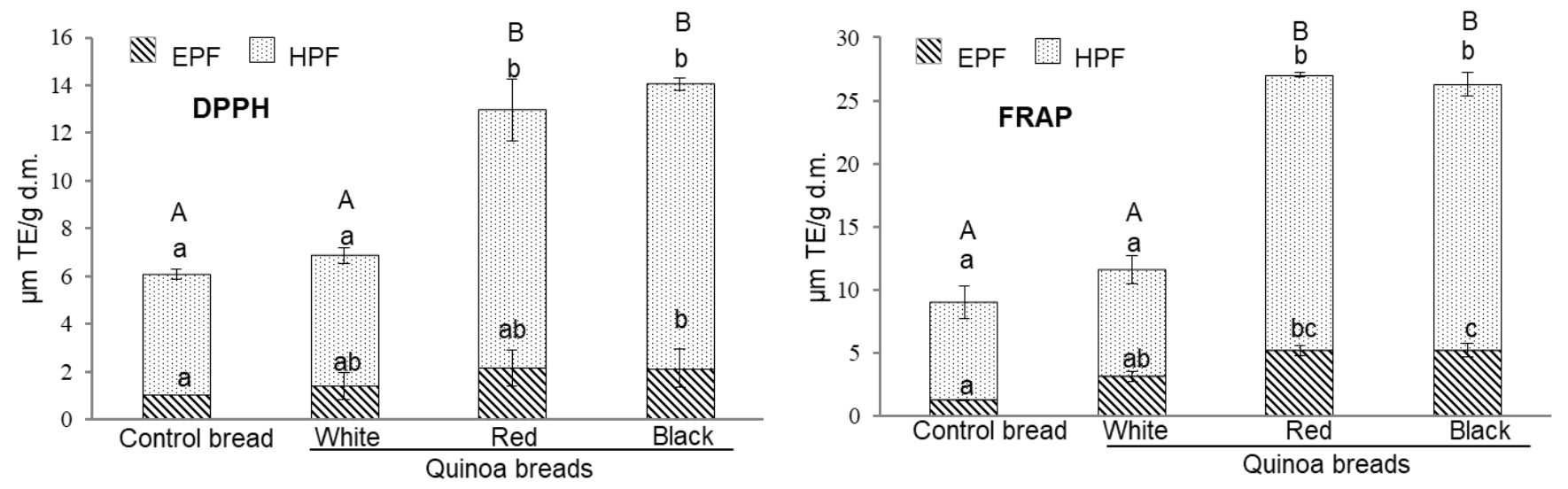

Fig. 2

\section{Electronic Supplementary Material}

ESM 1 Detailed description of Material and Methods

ESM 2 Table 1 Total phenolic content and antioxidant activity of flours

ESM 3 Table 2 Colour parameters of flours and seeds

ESM 4 Table 3 Total phenolic content (mg GAE/g d.m.) of breads

ESM 5 Table 4 Colour parameters of breads

ESM 6 Table 5 Effect of baking on the antioxidant activity of the 25\% quinoa breads 
ESM 1 Detailed description of Material and Methods

\section{Bread-making procedure}

The control bread dough formula consisted of wheat flour (450 g), dehydrated yeast (2.5 g/100 g flour basis), sodium chloride (1.6 g/100 g flour basis) and distilled water (up to optimum absorption, 500 Brabender Units, 58.0 g/100 g, flour basis, according to [1]). Wheat flour was replaced with $25 \%$ whole quinoa flour on flour basis to the bread dough formula (water absorption, $58.7 \mathrm{~g} / 100 \mathrm{~g}$ flour basis). Breads (in duplicated) were elaborated in a breadmaker (BM 3989, Severin, Germany) following the manufacturer indications. The pre-established baking program 1 and the strongest toasting level were used. Process variables consisted in the following steps: 1st kneading phase and rising phase for 9 min and 20 min respectively; 2nd kneading phase and rising phase for 14 min and 20 min respectively; short stirring for $30 \mathrm{sec}$; 3rd rising phase for 4 min and $30 \mathrm{sec}$; last rising phase for $45 \mathrm{~min}$ and lastly baking along $60 \mathrm{~min}$ at $170^{\circ} \mathrm{C}$. The obtained breads were dried at $40^{\circ} \mathrm{C}$ for $3 \mathrm{~h}$ by forced-air convection oven drying (Binder, Germany) and ground to a fine powder in a domestic mincer (Moulinex, France).

\section{Extraction of extractable and hydrolysable phenolic compounds}

The extractable polyphenols fractions (EPF) were obtained from $0.25 \mathrm{~g}$ of the flours (wheat and quinoa) and the ground breads in two consecutive 1-hour incubation steps at $24^{\circ} \mathrm{C}(10 \mathrm{ml}$ acidic methanol/water (50:50, v/v; $\mathrm{pH} 2$ ) and $10 \mathrm{ml}$ acetone/water (70:30, v/v)). The hydrolysable polyphenols fractions (HPF) were obtained by acidic hydrolysis done with the resulting residues using $20 \mathrm{~mL}$ of methanol and $2 \mathrm{~mL}$ of concentrated sulphuric acid (85 ${ }^{\circ} \mathrm{C}$ for $20 \mathrm{~h}$ ). Samples were then centrifuged (5000 g for $10 \mathrm{~min}$ ), washed two times with distilled water and finally taken to a final volume of $5 \mathrm{ml}$. EPF and HPF were stored at $-20^{\circ} \mathrm{C}$.

\section{Polyphenol content}


For PC determination, absorbance was measured at $724 \mathrm{~nm}$ in a microplate spectrophotometer reader (Power Wave HT, BioTek Instruments, Winooski, VT) and compared with a standard curve of Gallic acid (1, 0.70, 0.5, 0.25, 0.125, $0.0625 \mathrm{mM})$. Results were expressed as mg of Gallic Acid Equivalents (GAE) g-1 sample dry basis (DB). Determinations were performed per triplicate in each extract.

\section{Determination of antioxidant capacity}

DPPH assay: $50 \mu \mathrm{L}$ of diluted sample were mixed with a $250 \mu \mathrm{L}$ of DPPH methanolic solution (60 $\mu \mathrm{M})$ and incubated for 30 minutes at room temperature. Absorbance was read at $517 \mathrm{~nm}$. FRAP assay: $260 \mu \mathrm{L}$ of the FRAP reagent were mixed with $40 \mu \mathrm{L}$ of the diluted sample and incubated for 30 minutes at $37^{\circ} \mathrm{C}$. Absorbance was read at $593 \mathrm{~nm}$. Determinations were made in triplicate in each extract with a microplate spectrophotometer reader (Power Wave HT, BioTek Instruments, Winooski, VT, USA) and the results were expressed as $\mu$ mol of Trolox equivalents (TE)/g sample dry (d.m.).

\section{References}

[1] AACC (1995). Method 54-21: Farinograph method for flour. Saint Paul, Minnesota: Approved Methods of American Asociation of Cereal Chemistry. 
ESM 2 Table 1 Total phenolic content and antioxidant activity of flours

Table 1 Phenolic content and antioxidant activity of flours

\begin{tabular}{lrrrr}
\hline & \multirow{2}{*}{ Wheat } & \multicolumn{3}{c}{ Quinoa } \\
\cline { 3 - 5 } & & \multicolumn{1}{c}{ White } & \multicolumn{1}{c}{ Red } & \multicolumn{1}{c}{ Black } \\
\hline Phenolic content (mg GAE/g d.m.) & & & \\
EPF & $6.53 \pm 0.01 \mathrm{~b}$ & $5.03 \pm 0.46 \mathrm{a}$ & $5.13 \pm 0.22 \mathrm{a}$ & $6.60 \pm 0.46 \mathrm{~b}$ \\
HPF & $7.37 \pm 0.35 \mathrm{a}$ & $12.64 \pm 2.21 \mathrm{~b}$ & $23.07 \pm 0.34 \mathrm{c}$ & $24.10 \pm 1.84 \mathrm{c}$ \\
Total (EPF +HPF) & $13.90 \pm 0.36 \mathrm{a}$ & $17.67 \pm 2.26 \mathrm{a}$ & $28.20 \pm 0.41 \mathrm{~b}$ & $30.69 \pm 1.89 \mathrm{~b}$ \\
Antioxidant capacity ( $\mathbf{\mu m o l}$ TE/g d.m.) & & & \\
DPPH & & & & \\
EPF & $1.75 \pm 0.29 \mathrm{a}$ & $3.37 \pm 0.15 \mathrm{~b}$ & $8.28 \pm 0.39 \mathrm{c}$ & $9.30 \pm 0.00 \mathrm{~d}$ \\
HPF & $7.05 \pm 1.76 \mathrm{a}$ & $12.12 \pm 4.88 \mathrm{~b}$ & $23.46 \pm 1.18 \mathrm{c}$ & $21.18 \pm 0.91 \mathrm{c}$ \\
Total (EPF +HPF) & $8.80 \pm 1.76 \mathrm{a}$ & $15.50 \pm 4.88 \mathrm{a}$ & $31.74 \pm 1.24 \mathrm{~b}$ & $30.49 \pm 0.91 \mathrm{~b}$ \\
FRAP & & & & \\
EPF & $1.27 \pm 0.03 \mathrm{a}$ & $2.70 \pm 0.45 \mathrm{~b}$ & $10.73 \pm 0.13 \mathrm{c}$ & $13.30 \pm 0.02 \mathrm{~d}$ \\
HPF & $10.60 \pm 0.11 \mathrm{a}$ & $8.25 \pm 1.14 \mathrm{a}$ & $44.94 \pm 5.31 \mathrm{~b}$ & $39.64 \pm 0.83 \mathrm{c}$ \\
Total (EPF +HPF) & $11.87 \pm 0.12 \mathrm{a}$ & $10.95 \pm 1.22 \mathrm{a}$ & $55.67 \pm 5.31 \mathrm{~b}$ & $52.93 \pm 0.83 \mathrm{~b}$ \\
\hline
\end{tabular}

Codes: d.m. (dry matter), EPF (extractable polyphenols fraction), HPF (hydrolysable polyphenols fraction), FRAP (ferric reducing antioxidant power); DPPH ( $\alpha, \alpha$-diphenyl- $\beta$-picrylhydrazyl free radical scavenging method); GAE (gallic equivalents); TE (trolox equivalents)

Data expressed as mean \pm standard deviation. Means within lines followed by the same letter are not significantly different according to LSD post-hoc test at $99 \%$ confidence level 
ESM 3 Table 2 Colour parameters of flours and seeds

Table 2 Colour parameters of flours and seeds

\begin{tabular}{ccrrr}
\hline & \multirow{2}{*}{ Wheat } & \multicolumn{3}{c}{ QUINOA } \\
\cline { 3 - 5 } & & \multicolumn{1}{c}{ White } & \multicolumn{1}{c}{ Red } & \multicolumn{1}{c}{ Black } \\
\hline FLOURS & & & & \\
$\mathrm{L}^{*}$ & $89.9 \pm 1.8 \mathrm{~d}$ & $85.6 \pm 1.8 \mathrm{c}$ & $73.8 \pm 0.0 \mathrm{~b}$ & $69.7 \pm 0.8 \mathrm{a}$ \\
$\mathrm{a}^{*}$ & $-1.7 \pm 0.1 \mathrm{a}$ & $-1.5 \pm 0.2 \mathrm{a}$ & $2.1 \pm 0.0 \mathrm{c}$ & $0.9 \pm 0.1 \mathrm{~b}$ \\
$\mathrm{~b}^{*}$ & $10.1 \pm 0.3 \mathrm{a}$ & $13.1 \pm 0.8 \mathrm{c}$ & $12.6 \pm 0.0 \mathrm{c}$ & $11.4 \pm 0.2 \mathrm{~b}$ \\
$\Delta \mathrm{E}^{*}$ & - & $3.8 \pm 0.2 \mathrm{a}$ & $15.5 \pm 1.8 \mathrm{~b}$ & $19.9 \pm 2.6 \mathrm{~b}$ \\
$100-\mathrm{L}^{*}$ & $10.1 \pm 1.8 \mathrm{~d}$ & $14.4 \pm 1.8 \mathrm{c}$ & $26.2 \pm 0.0 \mathrm{~b}$ & $30.3 \pm 0.8 \mathrm{a}$ \\
SEEDS & & & & \\
$\mathrm{L}^{*}$ & n.d. & $76.2 \pm 1.4 \mathrm{c}$ & $38.2 \pm 1.3 \mathrm{~b}$ & $31.8 \pm 0.4 \mathrm{a}$ \\
$\mathrm{a}^{*}$ & n.d. & $0.2 \pm 0.3 \mathrm{a}$ & $13.0 \pm 0.4 \mathrm{c}$ & $6.5 \pm 0.6 \mathrm{~b}$ \\
$\mathrm{~b}^{*}$ & n.d. & $19.8 \pm 0.4 \mathrm{~b}$ & $18.0 \pm 0.9 \mathrm{~b}$ & $8.1 \pm 0.5 \mathrm{a}$ \\
\hline
\end{tabular}

$\Delta \mathrm{E}^{*}=\left[\left(\Delta \mathrm{L}^{*}\right)^{2}+\left(\Delta \mathrm{a}^{*}\right)^{2}+\left(\Delta \mathrm{b}^{*}\right)^{2}\right]^{1 / 2}$ (calculated with respect to wheat flour) n.d. (not determined, the wheat grains from which the commercial flour used was obtained were not available)

Data expressed as mean \pm standard deviation. Means within lines followed by the same letter are not significantly according to LSD post-hoc test at $99 \%$ confidence level 
ESM 4 Table 3 Total phenolic content (mg GAE/g d.m.) of breads

Table 3 Phenolic content (mg GAE/g d.m.) of breads

\begin{tabular}{lrrrr}
\hline & \multicolumn{4}{c}{ BREADS } \\
\cline { 2 - 5 } & \multicolumn{1}{c}{ Control } & White quinoa & \multicolumn{1}{c}{ Red quinoa } & Black quinoa \\
\hline EPF & $1.74 \pm 0.80 \mathrm{a}$ & $3.35 \pm 0.25 \mathrm{~b}$ & $3.89 \pm 0.01 \mathrm{~b}$ & $3.60 \pm 0.11 \mathrm{~b}$ \\
HPF & $17.19 \pm 0.23 \mathrm{a}$ & $17.34 \pm 0.05 \mathrm{a}$ & $20.23 \pm 2.99 \mathrm{a}$ & $19.82 \pm 0.05 \mathrm{~b}$ \\
Total (EPF + HPF) & $18.93 \pm 0.24 \mathrm{a}$ & $20.70 \pm 0.26 \mathrm{a}$ & $24.12 \pm 2.99 \mathrm{a}$ & $23.43 \pm 0.12 \mathrm{a}$ \\
\hline
\end{tabular}

Codes: d.m. (dry matter), EPF (extractable polyphenols fractions), HPF (hydrolysable polyphenols fractions), GAE (gallic equivalents)

Data expressed as mean \pm standard deviation. Means within lines followed by the same letter are not significantly different according to LSD post-hoc test at 99\% confidence level 
ESM 5 Table 4 Colour parameters of breads

Table 4 Colour parameters of breads

\begin{tabular}{lcccc}
\hline & \multicolumn{4}{c}{ BREADS } \\
\cline { 2 - 5 } & Control & White quinoa & Red quinoa & Black quinoa \\
\hline CRUST & & & & \\
$\mathrm{L}^{*}$ & $59.5 \pm 2.5 \mathrm{~b}$ & $49.2 \pm 2.7 \mathrm{a}$ & $47.1 \pm 4.2 \mathrm{a}$ & $45.3 \pm 3.5 \mathrm{a}$ \\
$\mathrm{a}^{*}$ & $7.2 \pm 1.1 \mathrm{a}$ & $11.3 \pm 0.3 \mathrm{~b}$ & $10.6 \pm 1.4 \mathrm{~b}$ & $9.4 \pm 1.0 \mathrm{ab}$ \\
$\mathrm{b}^{*}$ & $30.6 \pm 1.7 \mathrm{c}$ & $28.2 \pm 2.2 \mathrm{bc}$ & $24.9 \pm 0.5 \mathrm{ab}$ & $23.9 \pm 0.8 \mathrm{a}$ \\
$\Delta \mathrm{E}^{*}$ & - & $9.5 \pm 2.9 \mathrm{a}$ & $13.0 \pm 3.1 \mathrm{a}$ & $15.2 \pm 3.2 \mathrm{a}$ \\
$100-\mathrm{L}^{*}$ & $40.5 \pm 2.5 \mathrm{~b}$ & $50.8 \pm 2.7 \mathrm{a}$ & $52.9 \pm 4.2 \mathrm{a}$ & $54.7 \pm 3.5 \mathrm{a}$ \\
CRUMB & & & & \\
$\mathrm{L}^{*}$ & $69.1 \pm 2.2 \mathrm{~b}$ & $64.2 \pm 2.2 \mathrm{~b}$ & $50.8 \pm 2.0 \mathrm{a}$ & $46.3 \pm 1.0 \mathrm{a}$ \\
$\mathrm{a}^{*}$ & $-1.6 \pm 0.1 \mathrm{a}$ & $-1.3 \pm 0.1 \mathrm{a}$ & $4.6 \pm 0.4 \mathrm{c}$ & $2.9 \pm 0.2 \mathrm{~b}$ \\
$\mathrm{~b}^{*}$ & $14.9 \pm 1.3 \mathrm{ab}$ & $17.5 \pm 2.1 \mathrm{~b}$ & $15.5 \pm 0.3 \mathrm{ab}$ & $12.2 \pm 0.2 \mathrm{a}$ \\
$\Delta \mathrm{E}^{*}$ & - & $4.2 \pm 3.3 \mathrm{a}$ & $17.3 \pm 3.3 \mathrm{~b}$ & $22.8 \pm 2.1 \mathrm{~b}$ \\
\hline
\end{tabular}

$\Delta \mathrm{E}^{*}=\left[\left(\Delta \mathrm{L}^{*}\right)^{2}+\left(\Delta \mathrm{a}^{*}\right)^{2}+\left(\Delta \mathrm{b}^{*}\right)^{2}\right]^{1 / 2}$ (calculated with respect to control bread)

Data expressed as mean \pm standard deviation. Means within lines followed by the same letter are not significantly different according to LSD post-hoc test at $99 \%$ confidence level 
ESM 6 Table 5 Effect of baking on the antioxidant activity of the 25\% quinoa breads

Table 5 Effect of baking on the antioxidant activity of the 25\% quinoa breads

\begin{tabular}{|c|c|c|c|c|}
\hline & \multicolumn{2}{|c|}{ DPPH ( $\mu$ mol TE/g d.m.) } & \multicolumn{2}{|c|}{ FRAP ( $\mu$ mol TE/g d.m.) } \\
\hline & Flours $^{1}$ & Breads & Flours $^{1}$ & Breads \\
\hline \multicolumn{5}{|c|}{ Extractable polyphenol fraction (EPF) } \\
\hline WQ & $2.16 \pm 0.18 a$ & $1.41 \pm 0.55 a$ & $1.63 \pm 0.09 a$ & $3.13 \pm 0.41 b$ \\
\hline $\mathrm{RQ}$ & $3.38 \pm 0.12 \mathrm{a}$ & $2.17 \pm 0.75 a$ & $3.64 \pm 0.05 a$ & $5.15 \pm 0.39 b$ \\
\hline $\mathrm{BQ}$ & $3.64 \pm 0.22 a$ & $2.15 \pm 0.80 \mathrm{a}$ & $4.28 \pm 0.03 a$ & $5.20 \pm 0.55 a$ \\
\hline \multicolumn{5}{|c|}{ Hydrolyzable polyphenol fraction (HPF) } \\
\hline WQ & $8.32 \pm 2.54 a$ & $5.47 \pm 0.32 \mathrm{a}$ & $10.01 \pm 0.20 a$ & $8.47 \pm 1.12 a$ \\
\hline $\mathrm{RQ}$ & $11.15 \pm 1.03 a$ & $10.78 \pm 1.29 a$ & $19.19 \pm 1.41 a$ & $21.90 \pm 0.14 a$ \\
\hline $\mathrm{BQ}$ & $10.58 \pm 1.09 a$ & $11.89 \pm 0.25 a$ & $17.86 \pm 0.12 a$ & $21.08 \pm 0.93 b$ \\
\hline \multicolumn{5}{|c|}{ Total (EPF + HPF) } \\
\hline WQ & $10.47 \pm 2.50 a$ & $6.87 \pm 0.64 a$ & $11.64 \pm 0.22 a$ & $11.60 \pm 1.19 a$ \\
\hline $\mathrm{RQ}$ & $14.53 \pm 1.02 \mathrm{a}$ & $12.95 \pm 1.48 a$ & $22.82 \pm 1.41 a$ & $27.06 \pm 0.41 a$ \\
\hline $\mathrm{BQ}$ & $14.22 \pm 1.11 \mathrm{a}$ & $14.04 \pm 0.83 a$ & $22.14 \pm 0.12 a$ & $26.29 \pm 1.08 a$ \\
\hline
\end{tabular}

${ }^{1}$ Estimated by taking into account the percentage of each flour used to make doughs (75\% Wheat + 25\% Quinoa) and the corresponding antioxidant activity values in Table 1 "online resource”)

Codes: d.m. (dry matter), TE (Trolox equivalents), WQ (white quinoa), RQ (red quinoa), BQ (black quinoa)

Data expressed as mean \pm standard deviation. Letters in non-italic and italic refers to the comparisons of DPPH and FRAP determinations, respectively, between flour mixtures and breads. Means within lines followed by the same letter are not significantly different according to Student's $t$-test at the 95\% confidence level 